\title{
Metastable Changes to the Temperature Coefficients of Thin-film Photovoltaic Modules
}

\author{
Michael G. Deceglie, Timothy J Silverman, Bill Marion, Sarah R. Kurtz \\ National Renewable Energy Laboratory, Golden, Colorado, 80401, United States
}

\begin{abstract}
Transient changes in the performance of thin-film modules with light exposure are a well-known and widely reported phenomenon. These changes are often the result of reversible metastabilities rather than irreversible changes. Here we consider how these metastable changes affect the temperature dependence of photovoltaic performance. We find that in CIGS modules exhibiting a metastable increase in performance with light exposure, the light exposure also induces an increase in the magnitude of the temperature coefficient. It is important to understand such changes when characterizing temperature coefficients and when analyzing the outdoor performance of newly installed modules.
\end{abstract}

Index Terms-CIGS, photovoltaic, metastability, transient, light-soak, temperature coefficient

\section{INTRODUCTION}

Thin-film photovoltaic (PV) modules are known to exhibit a variety of transient and reversible changes in performance upon exposure to light [1]. In the case of CIGS devices, it is widely reported that both the fill factor $(F F)$ and open-circuit voltage $\left(V_{\mathrm{oc}}\right)$ increase with periods of exposure to sunlight, and that these changes reverse in the dark. The exact nature of such changes is known to depend on the particular formulation of the device layers. Here, we investigate how metastable changes to CIGS modules also affect the temperature coefficients of the performance parameters, i.e., the decrease in performance observed at elevated temperatures.

We observe that the slope of the power vs. temperature curve becomes steeper after prolonged light exposure. A variety of physical mechanisms are generally thought to contribute to transient reversible changes in CIGS modules, including interface and bulk trap states that change state under illumination [1]-[6]. In Section [II] we discuss how our results are consistent with light-induced interfacial barrier lowering. Our interpretation is based on the observation that change in the maximum power point $\left(P_{\mathrm{mp}}\right)$ temperature coefficient is driven by a change in the $F F$. It is also supported by a larger light-induced decrease in the apparent series resistance of the modules at low operating temperature compared to that at high operating temperature because electronic transport through or over an interfacial energy barrier is highly temperature dependent.

In general, the physics dominating different CIGS cells and modules can cause the devices to exhibit different lightinduced transient behavior. Previous work has investigated the possibility of light-induced changes to temperature coefficients, but no substantial effect was observed [7]. This highlights the importance of considering each product and CIGS formulation separately, because different devices can have different dominant physics, and thus, different performance changes due to illumination. It is critical to understand and account for light-induced changes when measuring temperature coefficients on new modules and when performing analysis of outdoor performance data for newly installed arrays to ensure that the outdoor performance of the module is appropriately captured.

\section{METHOD}

We conducted this study with two commercially available CIGS modules of the same model. Prior to this study the modules had not been exposed to light other than for initial characterization on a long-pulse solar simulator. The apparatus for the study was a light-soak chamber that uses an array of metal-halide lamps to illuminate the test plane at $1000 \mathrm{~W} \mathrm{~m}^{-2}$. The spatial variation in irradiance in the test plane was less than $5 \%$ and the irradiance was controlled with closed-loop feedback from an intensity sensor. The temperature of the modules was monitored with a thermocouple on the center of the back surface of the modules. We estimate the temperature measurement to be accurate within $\pm 2{ }^{\circ} \mathrm{C}$. Temperature was controlled with forced-air cooling based on closed-loop feed-

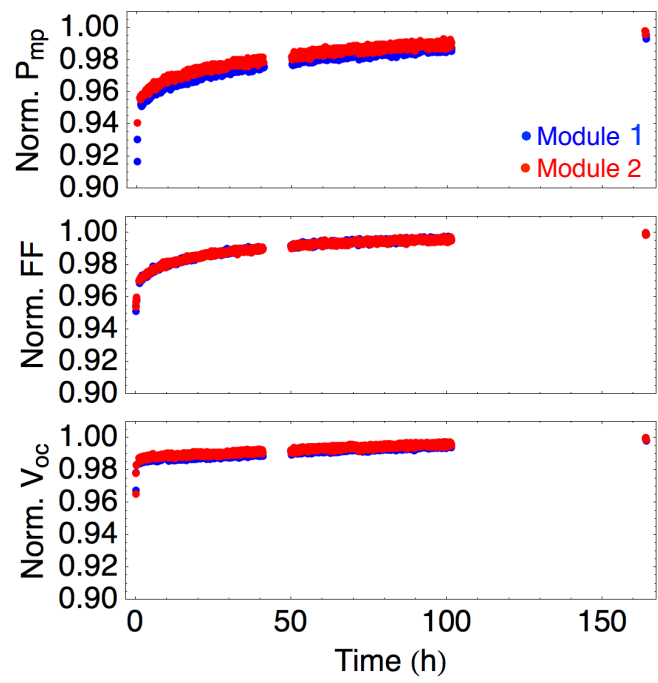

Fig. 1. Transient improvement in the $P_{\mathrm{mp}}, F F$, and $V_{\mathrm{oc}}$. The data are filtered for module temperatures between $53^{\circ} \mathrm{C}$ and $57^{\circ} \mathrm{C}$ to exclude the temperature ramps. Both $F F$ and $V_{\text {oc }}$ contribute to the improvement in $P_{\mathrm{mp}}$. 
back from the module thermocouples. For measurements taken in the light-soak chamber, we estimate a $\pm 0.25 \%$ uncertainty in the current and a $\pm 0.19 \%$ uncertainty in voltage.

We mounted the modules in the chamber at room temperature. The first stage of the test was a 30-minute warmup in which the lamps were struck and allowed to stabilize. The lamps remained on and controlled to $1000 \mathrm{~W} \mathrm{~m}^{-2}$ for the remainder of the test. During the warm-up, the module temperature stabilized at $55^{\circ} \mathrm{C}$. The module temperature was then ramped at a rate of $1 \mathrm{~K} \mathrm{~min}^{-1}$ from $55^{\circ} \mathrm{C}$ to $80^{\circ} \mathrm{C}$, then back to $55^{\circ} \mathrm{C}$. The module temperature was then maintained at $55^{\circ} \mathrm{C}$ for 163 hours. This 163 -hour phase is referred to as the light-soak phase for the purposes of this study. Data collection was lost for two periods during the 163-hour segment (apparent in Figure 1), but the illumination remained on, and the temperature controlled, during these intervals. After the 163-hour light soak, the same $1 \mathrm{~K} \mathrm{~min}^{-1}$ ramp from $55^{\circ} \mathrm{C}$ to $80^{\circ} \mathrm{C}$ was performed. We collected current-voltage (I-V) curves every five minutes during the test. The modules were biased at $P_{\mathrm{mp}}$ in the intervals between I-V curves.

To investigate the temperature coefficients of the performance parameters, namely $V_{\mathrm{oc}}, F F$, and $P_{\mathrm{mp}}$, we considered I-V curves collected during the ramps from $55^{\circ} \mathrm{C}$ to $80^{\circ} \mathrm{C}$ at the beginning and end of the test. To correct for the change in performance due to light exposure during the temperature ramps, we fit a line to the time series of $55^{\circ} \mathrm{C}$ data points just before and just after the ramp steps and use this fit to correct the slope of the performance parameter vs. temperature plots extracted from the ramp. We performed a linear fit to each of the performance vs. temperature data series, and normalized the plots to the the standard test condition parameter values provided on the manufacturer specification sheet. To eliminate noise in time-series $P_{\mathrm{mp}}$ data due to fluctuations in irradiance and uncertainty in the current measurement, the $P_{\mathrm{mp}}$ time series was linearly corrected by the ratio of the median short circuit current $\left(I_{\mathrm{sc}}\right)$ observed during the test to the measured $I_{\mathrm{sc}}$. The measured $I_{\mathrm{sc}}$ fluctuated less than $\pm 0.75 \%$.

To test whether the transient changes were reversible, we repeated the light-soak and temperature-coefficient measurement procedure after a period of dark storage, part of which was at elevated temperature. After 330.3 hours of total light exposure, the modules were stored at room temperature for approximately one week and then at $55^{\circ} \mathrm{C}$ for 165 hours. After an additional four days of dark storage at room temperature, the light-soak and temperature-coefficient measurement process was then repeated.

We also fit I-V curves with the single-diode model [8] to quantify the change in the shape of the characteristic. The fit was performed using a nonlinear optimization algorithm to first optimize the photocurrent $\left(I_{p h}\right)$, saturation current $\left(I_{p h}\right)$, series resistance $\left(R_{S}\right)$, and diode ideality factor $(n)$ while assuming a very high shunt resistance $\left(R_{s h}\right)$. These preliminary parameters were then used as input to a second-stage fit where the shunt resistance was allowed to vary along with the other parameters. For these I-V curves, we found that this approach was very robust to choice of starting parameters. To make the fit physically relevant, the measured voltages were divided by the number of cells in the series-connected module.

\section{RESUlts AND Discussion}

The modules used in this study exhibited a substantial improvement in performance with extended periods of light exposure. Time-series data showing the transient changes in $P_{\mathrm{mp}}, V_{\mathrm{oc}}$, and $F F$ at constant temperature $\left(55^{\circ} \mathrm{C}\right)$ are shown in Figure 11. This transient improvement occurred over more than 100 hours of continuous illumination. Both the $F F$ and $V_{\mathrm{oc}}$ show monotonic improvement but with different profiles in time.

TABLE 1

Temperature coefficients for $P_{\mathrm{mp}}$ and $F F$ measured at different stages of the experiment.

\begin{tabular}{|c|c|c|}
\hline Stage & $P_{\mathrm{mp}}$ Temp. Co. $\left(\% \mathrm{~K}^{-1}\right)$ & $F F$ Temp. Co. $\left(\% \mathrm{~K}^{-1}\right)$ \\
\hline Initial & -0.42 & -0.12 \\
Light soak & -0.46 & -0.16 \\
Dark storage & -0.44 & -0.14 \\
Additional light soak & -0.47 & -0.16 \\
\hline
\end{tabular}

Figure 2 compares the pre- and post-light-soak temperature dependence of the performance parameters. The associated temperature coefficients are summarized in Table 1 The values in Table 1 are the mean from the two modules, and are reported as a percentage of the performance parameter at $25^{\circ} \mathrm{C}$. For both modules, we observe a steeper slope for the post-light-soak normalized $P_{\mathrm{mp}}$ vs. temperature, indicating a change in the maximum-power temperature coefficient. This change corresponds to a relative increase in magnitude of the mean $P_{\mathrm{mp}}$ temperature coefficient of $9 \%$. As shown in Figure 2, the $V_{\mathrm{oc}}$ temperature coefficient does not change substantially upon light-soaking; the mean for the two modules remains relatively constant at $0.31 \% \mathrm{~K}^{-1}$. We observed the largest relative change in the $F F$ temperature coefficient, which increased in magnitude by $29 \%$.

Further insight can be gained from our experimental results by considering how the light soak changes the I-V characteristic at different temperatures. I-V curves collected at different temperatures before and after the light-soak phase are shown for module 1 in Figure 3 . The current and voltage values of the curves are normalized to the maximum and minimum values from the post-light-soak $55^{\circ} \mathrm{C}$ curve. The single-diodemodel fit parameters for the curves from Figure 3 are shown in Table 2. The results in Figure 3 show that the light soak improves the $F F$ more at low operating temperature than at high temperature. This result is also apparent in Figure 2. The parameters in Table 2 show that this change can be described by a larger light-induced decrease in series resistance at low temperature than at high temperature.

Both the $F F$ and $V_{\text {oc }}$ light-induced transients observed here have been widely observed. For a discussion regarding the physical origin of the changes in temperature-dependent performance, we focus on the change in $F F$ because the relative light-induced change in temperature coefficient is largest in $F F$. One mechanism frequently cited as contributing 
(a)

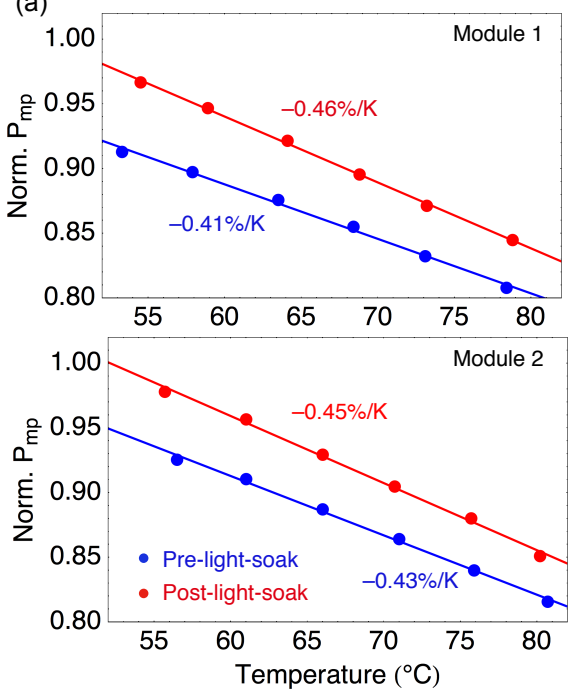

(b)
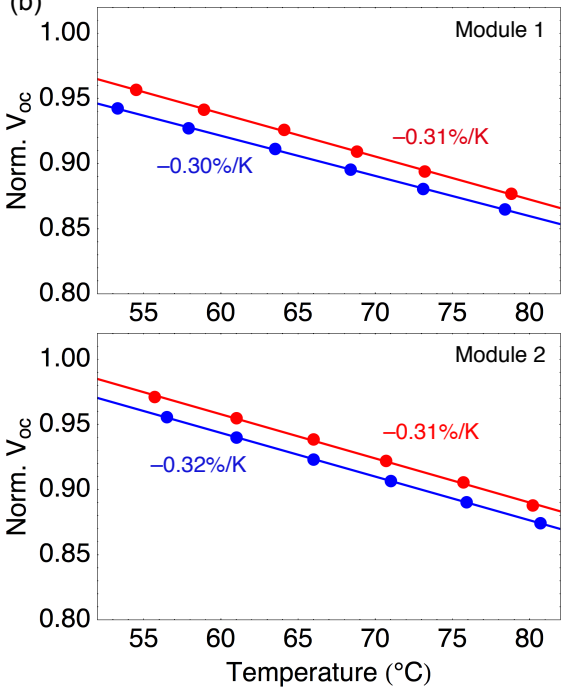

(c)
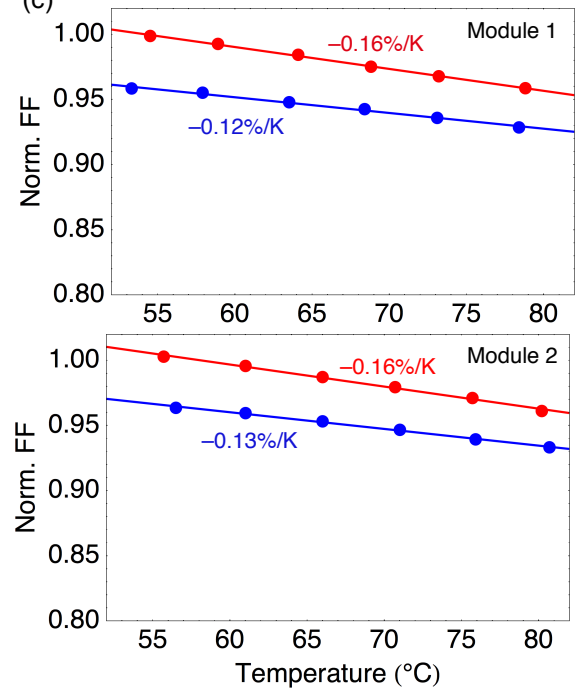

Fig. 2. The temperature dependence of $P_{\mathrm{mp}}, V_{\mathrm{oc}}$, and $F F$ measured on both modules by ramping the module temperature before ("pre-light-soak") and after ("post-light-soak") the first 163-hour light exposure. The magnitude of the $F F$ temperature coefficient increased by $29 \%$ upon light soaking. The parameters are normalized to the standard test condition values provided on the manufacturer specification sheet. The best fit-lines for each data set are also plotted and their slopes are noted on the plots.

TABLE 2

Single-diode-model fit parameters for the curves shown in Figure 3

\begin{tabular}{|ccccccc|}
\hline State & $\mathrm{Temp}^{\circ}{ }^{\circ} \mathrm{C}$ & $I_{p h}(\mathrm{~A})$ & $I_{o}(\mu \mathrm{A})$ & $R_{s}(\Omega)$ & $n$ & $R_{s h}(\Omega)$ \\
\hline Pre-light-soak & 53 & 2.24 & 0.2 & 0.035 & 1.34 & 6.84 \\
Pre-light-soak & 78 & 2.23 & 3.1 & 0.030 & 1.38 & 6.93 \\
Post-light-soak & 55 & 2.24 & 0.2 & 0.026 & 1.35 & 6.45 \\
Post-light-soak & 79 & 2.22 & 2.4 & 0.025 & 1.36 & 6.37 \\
\hline
\end{tabular}

to the transient behavior of CIGS modules is the energy barrier caused by the conduction-band offset between the buffer and the absorber [2]-[4]. Such a barrier can inhibit the collection of photo-excited carriers and thus may increase the apparent series resistance of the module. When the module is exposed to light, the electron and hole quasi-Fermi levels split, which drives deep level traps to change their charge state resulting in a reduction in the conduction band barrier. The conduction-band barrier inhibits the collection of photogenerated electrons, which must either tunnel through the barrier or undergo thermionic emission. When the barrier is reduced through light exposure, the transfer of electrons is enhanced, which improves the $F F$.

Our results are consistent with this microscopic picture of transient light-induced performance changes. Charge transfer across the conduction-band barrier, by tunneling or thermionic emission, is enhanced at elevated temperature. Therefore, a reduction in this barrier through light-soaking would be expected to benefit lower-temperature operation more. At lower operating temperature, the charge transfer across the barrier is less efficient; thus, performance benefits more from a reduction in this barrier. This explanation is consistent with our results. As shown in Figures 2 and 3 , we observed more improvement in the $F F$ at low temperature than at high temperature. The fit parameters in Table 2 show that this change can be described by changes in the series resistance.

We found that the observed transient, including the change in temperature coefficient, is at least partially reversible. Storage of the module in the dark at room temperature for approximately one week and then at $55^{\circ} \mathrm{C}$ for 165 hours resulted in a $5 \%$ decrease in $P_{\mathrm{mp}}$ from the light-soaked state, as measured with a flash solar simulator at $25.9 \pm 0.3^{\circ} \mathrm{C}$. Upon light-soaking, the mean temperature coefficient for $P_{\mathrm{mp}}$ increased in magnitude from $-0.44 \% \mathrm{~K}^{-1}$ to $-0.47 \% \mathrm{~K}^{-1}$.

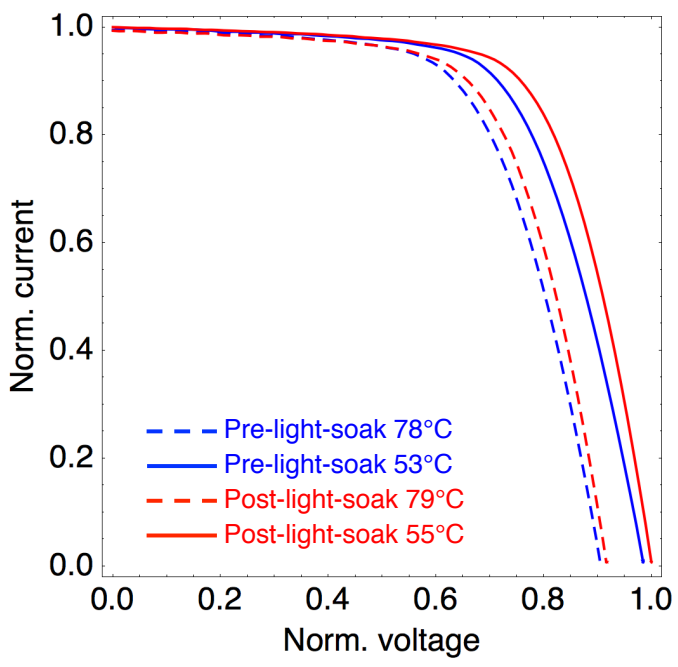

Fig. 3. Current-voltage curves collected for module 1 at the beginning and end of the first light exposure. The light exposure improves the lowtemperature $F F$ more than the high-temperature $F F$. 
This was driven by a change in the $F F$ temperature coefficient, with the mean temperature coefficient of $F F$ increasing in magnitude from $-0.14 \% \mathrm{~K}^{-1}$ to $-0.16 \% \mathrm{~K}^{-1}$, a $15 \%$ relative increase in the magnitude of the slope. The change in the temperature dependence of $F F$ is shown in Figure 4 These changes, summarized in Table 11, suggest that the changes in temperature coefficient are reversible, but we note that these changes are similar to the uncertainty of the measurement.

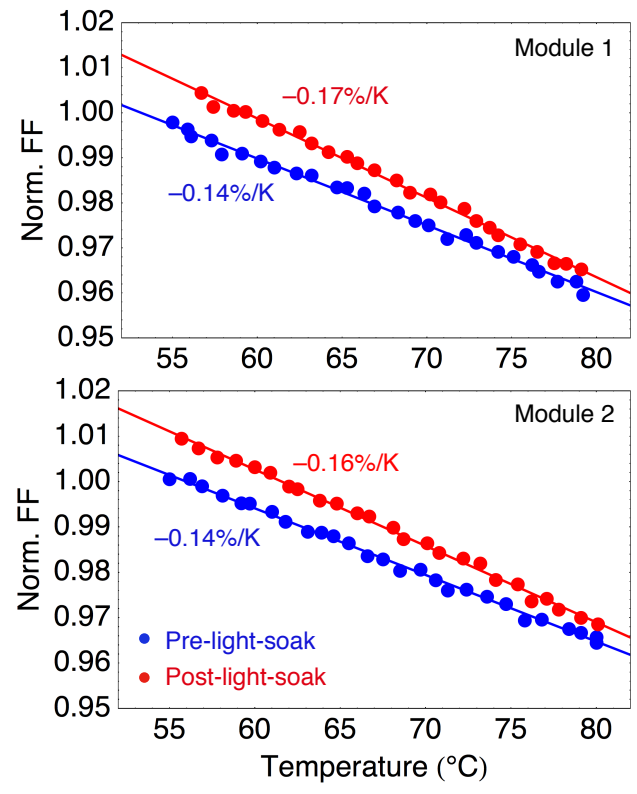

Fig. 4. The temperature dependence of $F F$ at the beginning and end of the final light-exposure. On average between the two modules, we observe a $15 \%$ increase in the magnitude of the slope upon light exposure. The $F F$ is normalized to that from the manufacture specification sheet. The best-fit lines for each data set are also shown and their slopes are indicated on the plot.

\section{CONCLUSION}

Here we have considered how metastable changes in CIGS modules affect not only the performance, but the degree to which performance changes with operating temperature. In particular, we observed a $29 \%$ increase in the magnitude of the $F F$ temperature coefficient upon light soaking, which results in a $9 \%$ increase in the magnitude of the the $P_{\mathrm{mp}}$ temperature coefficient. We suggest that this change is due to a light-induced reduction in the conduction-band barrier, which tends to benefit low-temperature performance more than high-temperature performance due to the thermally activated charge-transfer mechanisms governing forward-bias collection of photo-generated electrons. This suggests that the effect observed in this study would be sensitive to the electrically active interfaces in the device and would be expected to vary under different processing conditions. Ultimately, it is the final light-soaked temperature coefficient that will govern the photovoltaic output of an installation. Thus, it is critical to characterize and understand changes in the temperaturedependent performance due to light exposure when measuring temperature coefficients or applying temperature corrections to performance data from newly installed modules.

\section{ACKNOWLEDGMENT}

We gratefully acknowledge Steve Rummel, Allan Anderberg, and Keith Emery from NREL for I-V measurements that supported this work. We thank Kannan Ramanathan from NREL for insightful conversations. Some of the data in this report were obtained using equipment at the Energy Systems Integration Facility (a national user facility sponsored by the US DOE Office of Energy Efficiency and Renewable Energy) located at the National Renewable Energy Laboratory. This work was supported by the U.S. Department of Energy under Contract No. DE-AC36-08GO28308 with the National Renewable Energy Laboratory.

\section{REFERENCES}

[1] M. Gostein and L. Dunn, "Light soaking effects on photovoltaic modules: Overview and literature review," 2011 37th IEEE PVSC, pp. 003 126$003131,2011$.

[2] T. Kobayashi, H. Yamaguchi, and T. Nakada, "Effects of combined heat and light soaking on device performance of $\mathrm{Cu}(\mathrm{In}, \mathrm{Ga}) \mathrm{Se}_{2}$ solar cells with $\mathrm{ZnS}(\mathrm{O}, \mathrm{OH})$ buffer layer," Progress in Photovoltaics: Research and Applications, vol. 22, pp. 115-121, 2014.

[3] G. Agostinelli, E. D. Dunlop, D. L. Batzner, A. N. Tiwari, P. Nollet, M. Burgelman, and M. Kontges, "Light dependent current transport mechanisms in chalcogenide solar cells," in Photovoltaic Energy Conversion, 2003. Proceedings of 3rd World Conference on, vol. 1, 2003, pp. 356-359.

[4] M. Köntges, R. Reineke-Koch, P. Nollet, J. Beier, R. Schäffler, and J. Parisi, "Light induced changes in the electrical behavior of CdTe and $\mathrm{Cu}(\mathrm{In}, \mathrm{Ga}) \mathrm{Se}_{2}$ solar cells," Thin Solid Films, vol. 403-404, pp. 280-286, 2002.

[5] D. Dirnberger, "Uncertainty in PV module measurement-Part II: Verification of rated power and stability problems," Photovoltaics, IEEE Journal of, vol. 4, no. 3, pp. 991-1007, May 2014.

[6] S. Lany and A. Zunger, "Light- and bias-induced metastabilities in

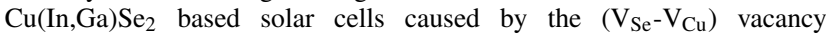
complex," Journal of Applied Physics, vol. 100, no. 11, pp. -, 2006. [Online]. Available: http://scitation.aip.org/content/aip/journal/jap/ 100/11/10.1063/1.2388256

[7] L. Dunn and M. Gostein, "Light soaking measurements of commercially available CIGS PV modules," in Photovoltaic Specialists Conference (PVSC), 2012 38th IEEE, June 2012, pp. 001 260-001 265.

[8] J. Nelson, The Physics of Solar Cells. World Scientific, 2003. 